For a bound electron, the last is not a Coulomb force, but is, rather, an elastic force $-\alpha r, r$ being the electron displacement. Writing the components in order, then, the total force is

$$
e \boldsymbol{E}_{0}+(4 \pi / 3) e P+0-\alpha r .
$$

At first sight it would appear that to make thi expression applicable to a free electron, it is only necessary to put $\alpha=0$. The presence of $(1 / 3) 4 \pi e P$ would then lead directly to a coefficient $3 / 2$ in (1).

But such a procedure neglects the Coulomb force of the ion which must replace the elastic binding force. This force can be calculated by supposing the positive ion to be uniformly distributed throughout a sphere of volume $1 / N$. The charge density is $N e$, so that when the electron. is displaced from the centre of this sphere, the restoring force is $-(4 \pi / 3) N e^{2} r$, and it is this which must replace $-\alpha r$. Since $P=N e r$, the total force on the free electron is seen to be

$$
e E_{0}+(4 \pi / 3) e P+0-(4 \pi / 3) e P=e E_{0} .
$$

Thus, when the field of the free positive ion replaces the molecular binding force, the polarisation term disappears as it should, since in fact no polarisation exists. It follows that the coefficient in the corresponding concentration equation is unity.

Research Laboratory,

General Electric Company, Schenectady, N.Y.

$$
\text { Oct. } 3 .
$$

I NATURE, 132, 101, July 15, 1933

1 Proc. Camb. Phil. Soc., 2\%, 143; 1933

\section{Stopping Layer of Rectifiers}

From measurements of the coefficient of rectification as a function of the thickness of the stopping layer, W. Jusé ${ }^{1}$ has deduced that rectification is only possible for a thickness of the stopping layer of about $10^{-5}-10^{-6} \mathrm{~cm}$. This is not in agreement with results

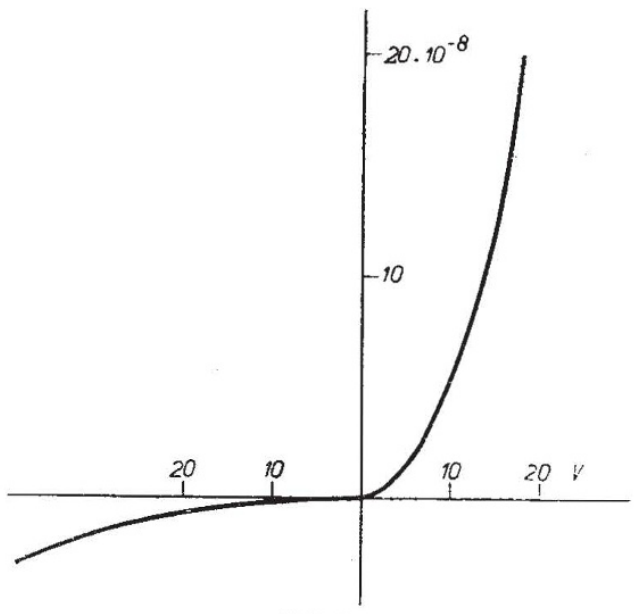

FIG. 1.

I have obtained with rectifiers, the stopping layers of which had thicknesses of $10^{-4}-10^{-3} \mathrm{~cm}$. The mean ratio of the currents in opposite directions varied with different samples and was about 10 , sometimes about 100 .

The rectifiers were made of a metal and a semiconductor, separated by a stopping layer, which can consist of every insulating material, for example, paper, artificial resin, the oxide of the base metal, etc. It is possible to get rectification with every thickness of the stopping layer between $10^{-6} \mathrm{~cm}$. (oxide-coated aluminium or zirconium) and $10^{-3} \mathrm{~cm}$. (condenser paper).

In Fig. 1 are given the characteristics of a rectifier with a stopping layer of $5 \times 10^{-4} \mathrm{~cm}$. thickness (amorphous $\mathrm{Al}_{2} \mathrm{O}_{3}$ on aluminium). The ratio of the currents in opposite directions is about 30 . In both directions the current can be represented by a function of the form $i=A V^{2}$.

So far as $I$ can see, these experiments are in agreement with the explanation of the rectifying process by cold emission ${ }^{2}$ and do not confirm the FrenkelJoffé-Nordheim-Wilson theory of the rectifying contact $^{3}$.

Natuurkundig Laboratorium der

W. Ch. vaN GeEL,

N. V. Philips' Glœilampenfabrieken, Eindhoven.

Oct. 10

2. Nature, 132, 242, Aug. 12, 1933.
W. Ch. v. Geel, Z. Phys., B9, 765; 1931.

${ }^{3}$ See R. H. Fowler, Ph. Z. Sovj. Un., 3, $526 ; 1933$.

\section{Developmental Anomalies in the Wistar Albino Rat} (Edinburgh Stock)

Is view of Ornsteen's suggestions ${ }^{1}$ offered in explanation of the Laurence-Biedl syndrome, it is of interest to record the occurrence of a group of peculiar developmental anomalies in the albino rat (Wistar derivatives, Edinburgh stock).

In one line of this stock several cases of microph. thalmia have been encountered. This eye defect, which has already been reported in the American stock by Addison ${ }^{2}$ and Danforth ${ }^{3}$, is not infrequently accompanied by opacity of the lens and blindness. In the typical case the eye is very small-roughly a sixth of the normal size-and pale pink ; the animals are, in other respects, fully representative of the stock. The distribution of this abnormality indicates that the character is genetic and most probably recessive.

In families related to the above but in which microphthalmia is not present, other peculiarities have been observed. Unilateral cryptorchidism has been found in three males, one of whom is the son of a microphthalmic male; seven females, sisters, possessing rudimentary male external genitalia in varying degrees of development are cousins of the oldest microphthalmic male in the stock. Another cousin has a cloaca instead of a normal rectal opening, together with the deeply cleft clitoris and the rudimentary penis present in the other females mentioned. A brother of the seven females alluded to has a son with a skull deformity which involves the loss of an eye. Within the group, also, there is a number of rats that have experienced difficulty in parturition, death supervening in several instances.

It seems not unreasonable to regard these various abnormal conditions as being instances of the polymorphic expression of one and the same genetic character.

\footnotetext{
Institute of Animal Genetics, King's Buildings, Edinburgh,

'Ornsteen, Amer. J. Med., 133, 256; 1932.

2 Addison, Anat. Rec., 20,344; 1925.

${ }^{3}$ Danforth, Amer. J. Anat., 45, 275; 1930.
}

A. M. HAIN. 